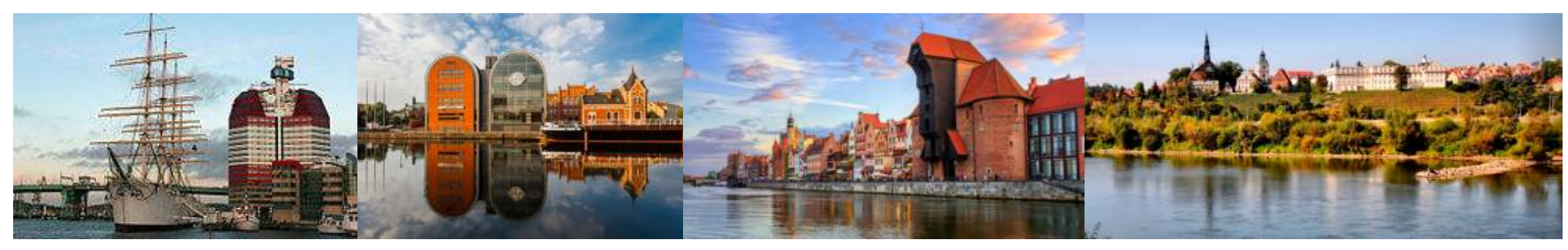

\title{
FACTORS FOR DELAYED CALL FOR EMERGENCY MEDICAL SERVICES IN ACUTE STROKE PATIENTS IN COMMUNITY HOSPITALS - DATA FROM POMERANIAN STROKE REGISTRY
}

\section{G. M. Kozera ${ }^{1}$, W. Szczuchniak², P. Sobolewski², W.M.Nyka ${ }^{3}$ on behalf of Pomeranian Stroke Registry collaborators}

${ }^{1}$ Collegium Medicum in Bydgoszcz, Nicolaus Copernicus University in Toruń, Department of Neurology, Bydgoszcz, Poland.

2 Department of Neurology and Stroke Unit, Holy Spirit Specialist Hospital, Sandomierz,

${ }^{3}$ Department of Neurology, Medical University of Gdańsk, Gdańsk,

Objectives: Delayed call for emergency medical services commonly reduce the efficacy of acute stroke treatment in Polish stroke centers due to prolongation of onset to door time. Poor recognition of stroke symptoms is one of the most commmon, but not the only reson for pre-hospital delays. Thus, the determination of factors influencing delayed call for ambulance in routine practice may help both, patients education and EMS logistics.

Aim: To evaluate presence of delayed call for ambulance among acute stroke patients treated at the community-based Polish stroke centers and to determine logistic, social, epidemiological, and clinical factors responsible for delayed call for EMS.

Materials and methods: This study carried out an evaluation of the medical records of 8710 patients with stroke $(90,25 \%$ : $80,94 \%$ ischemic and 9,30\% hemorrhagic land transient ischemic attack $/$ TIA $/(9,75 \%)$ from Polish stroke centers that have been consecutively reporting to the Pomeranian Stroke Registry from 2010 to 2013.

Table 1. Social, clinical and logistic factors in patients with immediate and daleyed call for ambulance.

\begin{tabular}{|c|c|c|c|}
\hline & $\begin{array}{c}\text { immediate }(<45 \mathrm{~min}) \\
\text { call for ambulance } \\
36,67 \% \\
\end{array}$ & $\begin{array}{c}\text { delayed (> } 45 \mathrm{~min} \text { ) } \\
\text { call for ambulance } \\
63,32 \%\end{array}$ & \\
\hline & $\mathrm{N}(\%)$ & $N(\%)$ & \\
\hline Male gender & $1787(49,45)$ & $3028(48,53)$ & 0,38 \\
\hline Ischaemic stroke & $2831(78,33)$ & $5145(82,45)$ & $<0,001$ \\
\hline Haemorrhagic stroke & $382(10,57)$ & $430(6,89)$ & $<0,001$ \\
\hline SAH & $59(1,63)$ & $46(0,74)$ & $<0,001$ \\
\hline TIA & $342(9,46)$ & $619(9,92$ & 0,46 \\
\hline mRS 0-2 & $1912(59,58)$ & $3923(66,59)$ & $<0,001$ \\
\hline Median age (IQR) [yrs] & $73(62-81)$ & $72(62-80)$ & 0,03 \\
\hline Age over 80 years & $889(25,58)$ & $1424(23,81)$ & 0,05 \\
\hline Regular physician's care prior stroke & $2302(63,70)$ & $3600(57,69)$ & $<0,001$ \\
\hline Retired/Pensioner & $2850(78,86)$ & $5081(81,43)$ & 0,002 \\
\hline Primary education (not high) & $2357(89,89)$ & $4031(89,78)$ & 0,87 \\
\hline Living alone $\mathbf{N}$ & $408(11,29)$ & $919(14,73)$ & $<0,001$ \\
\hline Hospital located in urban area (>100.000 inhabitants) & $1376(38,07)$ & $2144(34,36)$ & $<0,001$ \\
\hline Risk factors N (\%) & --- & --- & $-\cdots$ \\
\hline Hypertension & $2490(68,90)$ & $4313(69,12)$ & 0,82 \\
\hline Diabetes mellitus & $814(22,52)$ & $1481(23,73)$ & 0,17 \\
\hline Coronary heart disease & $1168(33,40)$ & $1656(27,37)$ & $<0,001$ \\
\hline Dyslipidaemia & $991(27,42)$ & $1674(26,83)$ & 0,52 \\
\hline Atrial fibrillation & $863(24,00)$ & $1057(17,02)$ & $<0,001$ \\
\hline Current smoker & $552(17,52)$ & $1182(21,55)$ & $<0,001$ \\
\hline Previous stroke or TIA & $1039(29,68)$ & $1641(27,30)$ & 0,01 \\
\hline New alcohol & $64(1,77)$ & $159(2,55)$ & 0,01 \\
\hline Pre-stroke anticoagulants & $283(7,87)$ & $352(5,67)$ & $<0,001$ \\
\hline Signs and symptoms of stroke & --- & --- & $-\cdots$ \\
\hline Headache & $955(26,43)$ & $958(15,35)$ & $<0,001$ \\
\hline Cognitive impairment & $1054(29,16)$ & $1154(18,49)$ & $<0,001$ \\
\hline Motor deficit/Paresis & $2643(73,13)$ & $4177(66,94)$ & $<0,001$ \\
\hline Dysphasia/Aphasia & $1644(45,49)$ & $2218(35,34)$ & $<0,001$ \\
\hline Sensory impairment & $587(16,24)$ & $981(15,72)$ & 0,49 \\
\hline Visual disturbances & $364(10,07)$ & $582(9,33)$ & 0,23 \\
\hline Seizures & $386(10,68)$ & $1118(17,92)$ & $<0,001$ \\
\hline Gait disturbances & $97(2,68)$ & $190(3,04)$ & 0,30 \\
\hline Referral to the hospital by EMS & $2736(75,71)$ & $3415(54,73)$ & $<0,001$ \\
\hline Refferal from GP & $229(6,34)$ & $1725(27,64)$ & $<0,001$ \\
\hline Pre stroke mRS 0-2 pts & $3181(88,02)$ & $5562(89,13)$ & 0,09 \\
\hline Median NIHSS score on admission (IQR) [pts] & $8(4-15)$ & $5(2-9)$ & $<0,001$ \\
\hline NIHSS 0-5 [pts] & $1286(36,48)$ & $3293(53,32)$ & $<0,001$ \\
\hline NIHSS 6-25 [pts] & $2138(60,65)$ & $2794(45,24)$ & $<0,001$ \\
\hline NIHSS >25 [pts] & $101(2,87)$ & $89(1,44)$ & $<0,001$ \\
\hline Received thrombolitic treatment & $436(12,06)$ & $35(0,56)$ & $<0,001$ \\
\hline
\end{tabular}
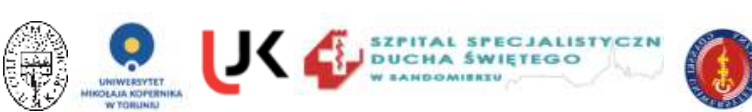

Results: Delayed call for emergency medical services (>45 min from symptoms onset) was reported $n$ 63.33\% of patients. Patients with delayed call for EMS were characterized by younger age (72 vs 73yrs; $p=0,03)$, higher presence of ischemic etiology $(82,4$ vs $78,3 \%, p<0,001)$, retired $(81,4$ vs $78,8 \% ; p=0,002)$ and living alone $(14,7$ vs $11,3 \% ; p<0,001)$, smokers $(21,5$ vs $17,5 \% ; p<0,001)$, chronic alcohol abusers $(2,5$ vs $1,8 \% ; p=0,01)$ and with seizures at stroke onset $(17,9$ vs $10,7 \% ; p<0,001)$. They were also characterized by lower NIHSS on admission ( 5 vs 8 pts, $p<0,001$ ) and lower presence of regular physician's care prior stroke $(57,7$ vs $63,7 \%$; $p<0,001)$, urban location $(34,4$ vs $38,1 \%, p<0,001)$, coronary heart disease $(27,4$ vs $33,4 \% ; p<0,001)$, atrial fibrillation $(17,0$ vs $24,0 \%$; $p<0,001)$, pre-stroke anticoagulants $(5,7$ vs $7,9 \%$; $\mathrm{p}<0,001)$, previous stroke or TIA $(27,3$ vs $29,9 \% ; p=0,01)$, headache $(15,3$ vs $26,4 \%$; $p<0,001)$, motor impairment $(66,9$ vs $73,1 \%$ )(Table 1 ).

Multivariate analysis showed that age, living alone, smoking and tobacco use, seizures, headache and speech deficits, urban location, regular physician's care prior stroke and presence of atrial fibrillation contributed toward delayed call for EMS (Figure 1).

Figure 1. Multivariate analysis toward delayed call for EMS.

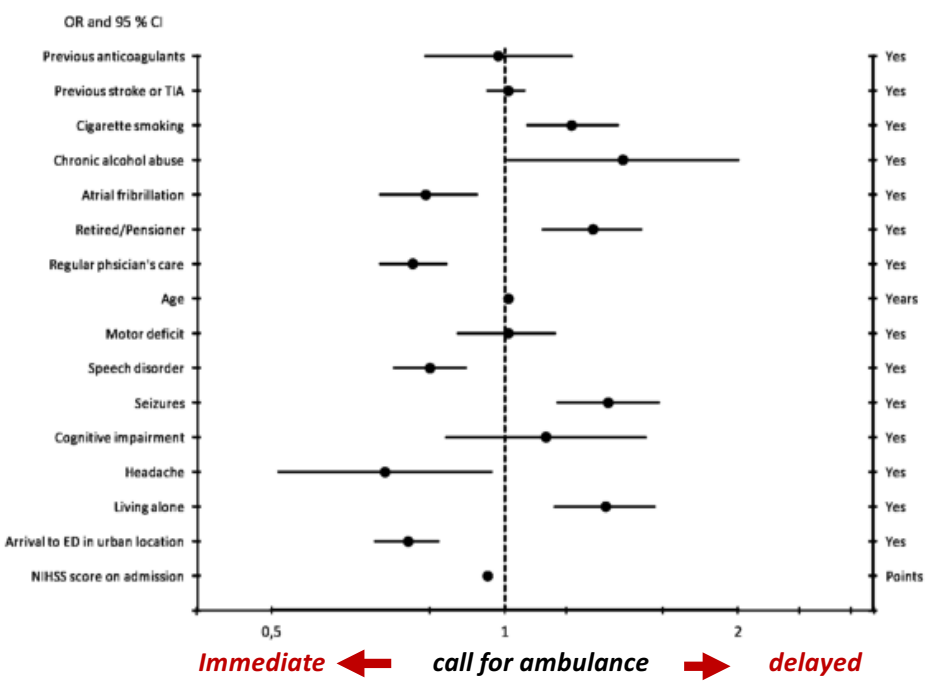

Conclusion: Delayed call for commonly exists among patients with stroke and transient ischemic attack due to numerous social, clinical and logistic factors. Educational campaign targeting high risk, living alone and rural located patients is necessary. 